\title{
Active longitudes, nonaxisymmetric dynamos and phase mixing
}

\author{
S. V. Berdyugina ${ }^{1,2}$, D. Moss ${ }^{3}$, D. Sokoloff ${ }^{4}$, and I. G. Usoskin ${ }^{5}$
}

\author{
${ }^{1}$ Institut für Astronomie, ETH Zentrum, 8092 Zürich, Switzerland \\ e-mail: sveta@astro.phys.ethz.ch \\ 2 Astronomy Division, PO Box 3000, 90014 University of Oulu, Finland \\ 3 School of Mathematics, University of Manchester, Oxford Road, Manchester M13 9PL, UK \\ 4 Department of Physics, Moscow State University, 119992 Moscow, Russia \\ 5 Sodankylä Geophysical Observatory (Oulu unit), 90014 University of Oulu, Finland
}

Received 17 May 2005 / Accepted 31 August 2005

\begin{abstract}
We discuss the problem of solar active longitudes from the viewpoint of dynamo theory. We start from a recent observational analysis of the problem undertaken by Berdyugina \& Usoskin (2003, A\&A, 405, 1121) and Usoskin et al. (2005, A\&A, 441, 347) who demonstrated from a study of sunspot data that solar active longitudes rotate differentially, with a small but significant asynchrony between northern and southern hemispheres. We suggest two concepts by which the underlying magnetic structure could lead to the observed phenomenology the true differential rotation of a nonaxisymmetric magnetic structure and a stroboscopic effect. In the latter case, a solid body rotation of nonaxisymmetric magnetic structure is illuminated by an activity wave propagating from middle latitudes to the solar equator, and so mimics a differential rotation. We then discuss several mechanisms which could in principle lead to the excitation of active longitudes. In particular, we consider dynamo excitation of nonaxisymmetric magnetic modes, nonaxisymmetric structures as a manifestation of a relic magnetic field in the solar core, nonaxisymmetric solar hydrodynamics and nonlinear instabilities that lack axial symmetry. We conclude that these mechanisms all provide ways to explain the phenomenology, provided the stroboscopic interpretation is accepted. Of course, a quantitative explanation in the framework of any scenario requires ultimately a detailed numerical simulation. The interpretation of the available observations as a true differential rotation appears to provide a much more severe challenge for theorists. We are unable to suggest a plausible mechanism of this kind; however we can not exclude in principle such an explanation. We relate the phenomenon of solar active longitudes to the information available concerning stellar active longitudes, and also consider evidence from other tracers of solar activity.
\end{abstract}

Key words. Sun: activity - Sun: magnetic fields - Sun: rotation - stars: magnetic fields - magnetic fields

\section{Introduction}

It has become clear that any proposed connection between dynamo theory and the occurrence of preferred longitudes in solar and stellar activity needs clarification. Many attempts have been undertaken to find preferred longitudes in solar activity from sunspot data (see e.g. Chidambara 1932; Lopez Arroyo 1961; Warwick 1965; Vitinskij 1969; Balthasar \& Schüssler 1983), as well as in other tracers of solar activity such as solar flares, the solar wind, etc. (e.g. Bumba \& Obridko 1969; Stewart\& Bravo 1996; Jetsu et al. 1997; Benevolenskaya et al. 1999a; Neugebauer et al. 2000). Preferred longitudes were often found over short time-scales, especially near the minimum of the solar activity. For instance, Benevolenskaya et al. (1999b) showed that the replacement of old magnetic flux with new flux of the opposite polarity during the transition from solar cycle 22 to 23 occurred within a narrow range of active longitudes. A recent analysis of the observational situation was undertaken by Berdyugina \& Usoskin (2003, and references therein) and Usoskin et al. (2005). In the first paper, an analysis of sunspot data was made with appropriate filtering techniques, leading to an identification of persistent active longitudes and their properties at a high significance level. This approach was criticised by Pelt et al. (2005) who expressed doubts about the reality of the active longitudes as revealed by the analysis. In a second paper Usoskin et al. (2005) analysed the sunspot distribution without any filtering and averaging, and confirmed the previous conclusions in a more robust and straightforward way.

A general conclusion based on these papers is that the solar activity is not a perfectly axisymmetric phenomenon and that some preferred centres of the activity or active longitudes do exist. These longitudes can be thought of as being where sunspots preferentially are formed (or, perhaps more accurately, first appear). The level of the nonaxisymmetry is low and it can be clearly found only in the statistical sense. Also these active longitudes follow the differential rotation rather than rotating with a constant rate, as would be intuitively expected.

The standard explanation of the solar activity cycle in terms of solar dynamo theory is based on what are basically axisymmetric models for the large-scale fields. The point is that the 
conventional dynamo mechanism for solar activity involves two magnetic field generators, namely the radial shear of differential rotation and the helicity of convective eddies associated with the " $\alpha$-effect". Both generators are normally considered as axisymmetric and a naive expectation is that the result of their action should be axisymmetric as well. (In fact, this expectation is not altogether true - in certain circumstances nonaxisymmetric fields can result from axisymmetric generators.) This is perhaps why the problem of active longitudes has not attracted more attention in the context of solar dynamo theory. Of course, the assumption of nonaxisymmetric solar hydrodynamics provides additional options to explain active longitudes. The quantitative explanation remains however nontrivial even in this case because differential rotation in the solar convective zone smooths longitudinal inhomogeneities of magnetic field.

Of course, there have been some attempts to address the topic of such preferred longitudes in stellar dynamo models (see e.g. Rädler et al. 1990; Moss et al. 1991a, 1995; Barker \& Moss 1994; Moss 2004, 2005; Bigazzi \& Ruzmaikin 2004) and also in more solar-like models (see e.g. Moss 1999; also Elstner \& Korhonen 2005). Analysis of stellar data introduces a number of new aspects into the problem of active longitudes. We briefly discuss this in a separate section. In any case, the existence of active longitudes on stars other than the Sun requires careful appraisal of dynamo models that are designed primarily to explain the solar case.

At this preliminary stage of our knowledge, a reasonable approach to the problem is to assemble a comprehensive description of the phenomenon of active longitudes and then to discuss a minimal set of theoretical ideas that might provide an explanation of the phenomenon. This is the aim of this paper. We believe that such an analysis can be more immediately relevant than the detailed development of a particular new model of nonaxisymmetric magnetic structure supported by a stellar dynamo mechanism.

The paper is organized as follows. We present a description of the active longitude phenomenon as recorded in sunspot data in Sect. 2. This picture assembles the features of nonaxisymmetric magnetic structures described in previous papers. We argue that these features effectively constrain possible explanations of the phenomenon in the framework of dynamo theory. A connection of this description with the language of dynamo theory is given in Sect. 3. In Sect. 4 we discuss some existing dynamo explanations for nonaxisymmetric solar magnetic structures. Section 5 presents a brief discussion of stellar active longitudes. Our conclusions are summarized in Sect. 6.

\section{Active longitudes as recorded in sunspot data}

An essential problem in the study of active longitude is related to the differential rotation of the solar surface, which makes it difficult to link an observed instantaneous location on the disc to a fixed longitude. Since the latitude of the solar magnetic activity changes in the course of a solar cycle, the observed instantaneous longitudes must be normalized with respect to the differential rotation. The usual normalization is related to the so-called Carrington longitudes ${ }^{1}$, assuming a fixed rotation rate as defined from sunspots. However, we can not a priori accept the Carrington rotation rate for active longitudes since its definition is not based on the concept of active longitudes. Berdyugina \& Usoskin (2003) have performed a systematic analysis of the occurrence of sunspots since the 1870 s without a priori assumption about a fixed rotation rate of active longitudes. They have shown that there are two persistent active longitudes $180^{\circ}$ apart whose longitudinal migration is defined by the differential rotation and the mean latitude of sunspot formation, while the Carrington rotation law smears this pattern on time-scales of a solar cycle. Usoskin et al. (2005) have shown that active longitudes are persistent in a dynamical reference frame which is not associated with a uniform rotation, but rotates differentially. This differential rotation is the effect of the equatorial migration of the sunspot "belt", which causes the nascent spots to experience a faster local rotation rate as the solar cycle proceeds. This is illustrated in Fig. 1 which shows the two semi-annually averaged active longitudes (crosses) defined from actual sunspot data.

In the absence of differential rotation the two migration paths would be linear, and the slope of the paths would indicate the difference between the Carrington period and the true period of migration. If the mean period related to the active longitudes is longer than the Carrington period, the active longitude will lag behind the Carrington meridian, and the lag will increase with time. In the opposite case, the active longitude will overtake the Carrington meridian with the difference in longitude also accumulating over time.

Deviations from a linear migration imply that the period of migration is a function of time. The "cycloid" shape of the paths is a clear signature of the differential rotation: the lag in longitude is increasing with respect to the Carrington frame at the beginning of the cycle when sunspots appear at high, more slowly rotating latitudes and then starts to decrease as the sunspot belt approaches the more rapidly rotating equator. The migration pattern has a clear 11-year periodicity, corresponding to the 11-year sunspot cycle.

In the general case the lag in longitude accumulated over a time period $\Delta T_{i}$ can be expressed as

$\Delta \Lambda_{i}=\left(\Omega_{\mathrm{C}}-\Omega_{i}\right) \Delta T_{i}$,

where $\Omega_{\mathrm{C}}$ is the Carrington angular velocity and $\Omega_{i}$ is the mean angular velocity of the active longitude during the period $\Delta T_{i}$.

\footnotetext{
1 Lord Carrington determined the solar rotation rate by observing low-latitude sunspots in the 1850s. He defined a fixed solar coordinate system that rotates in a sidereal frame exactly once every 25.38 days (Carrington 1863, pp. 221, 244). The synodic rotation rate varies a little during the year because of the eccentricity of the Earth's orbit. The mean synodic value of 27.2753 days is used to define the Carrington synodic system. As the original prime meridian Carrington selected the meridian that passed through the ascending node of the Sun's equator at 12:00 UT on 1 January 1854. The sidereal period is the solar rotation period relative to the stars. The synodic period is the time that it takes for the prime solar meridian to reappear at the same place in the sky as observed from Earth. The synodic and sidereal periods differ because the Earth itself revolves around the Sun.
} 

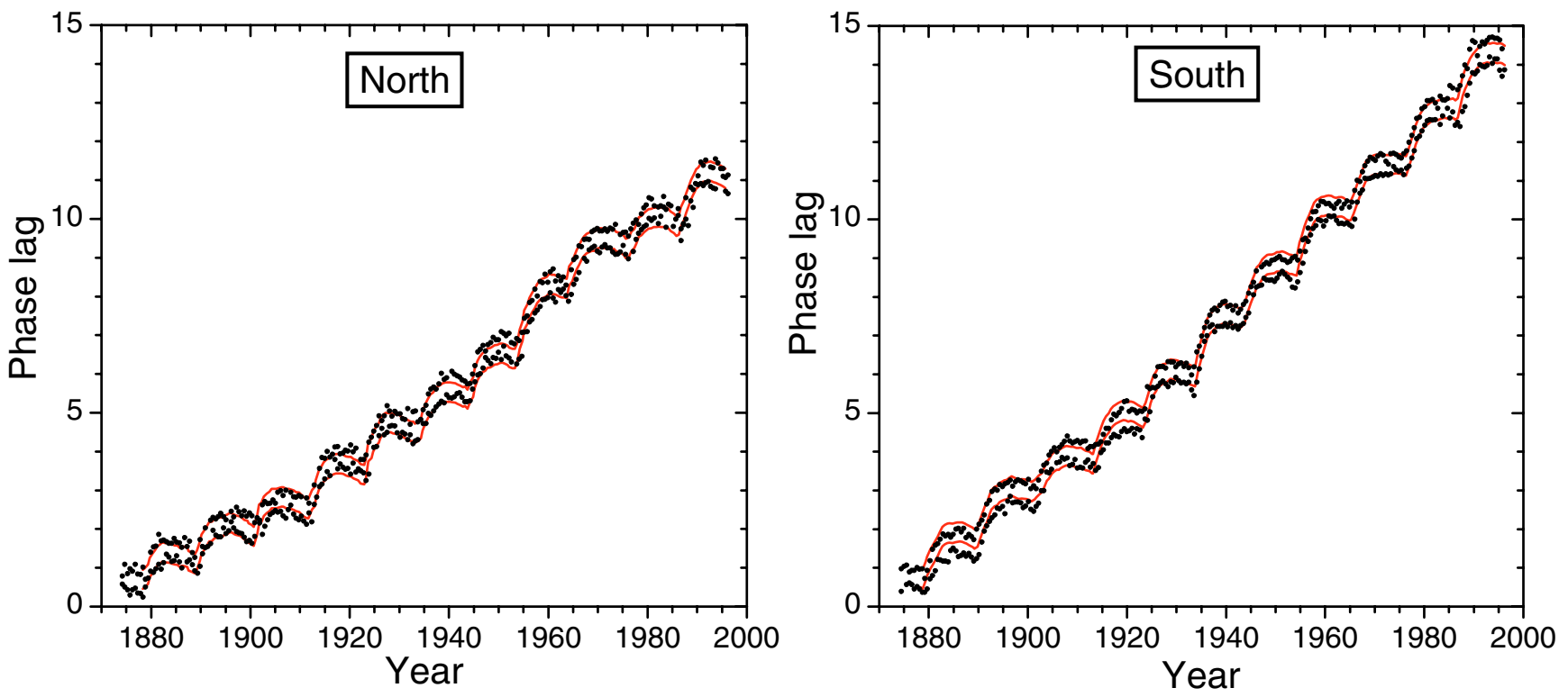

Fig. 1. Phase lags $\left(\Delta \Lambda / 360^{\circ}\right)$ of the semi-annual averaged longitudes of the two pronounced centres of sunspot activity (dots). Integers were added to the fractional phases in order to display the migration as a continuous curve (see Berdyugina \& Usoskin 2003). Lines depict the migration paths of the two active longitudes calculated from the best fit parameters of the differential rotation and mean latitude of sunspot occurrence, according to Eqs. (1) and (2). Data for the Northern and Southern hemisphere are analysed separately. The "cycloid" shape of the paths is a signature of the differential rotation: the lag in longitude is increasing with respect to the Carrington frame in the beginning of the cycle when sunspots appear at high, slower rotating latitudes and then start decreasing as the sunspot belt approaches the more rapidly rotating equator. Because of the 11-year cycle in sunspot latitudes, the migration pattern has a clear 11-year periodicity.

As mentioned above, if $\Omega_{i}=$ const., the migration path is linear. In the presence of differential rotation, $\Omega_{i}$ is a function of latitude, which is often approximated as

$\Omega_{i}=\Omega_{0}-\Delta \Omega \sin ^{2} \psi_{i}$,

where $\Delta \Omega$ is a rotational shear between the pole and the equator, or differential rotation rate, $\psi_{i}$ is a mean latitude of the tracer over the time period $\Delta T, \Omega_{0}$ is the angular velocity at the equator. Knowing $\psi_{i}$ and $\Delta \Lambda$, the parameters $\Omega_{0}$ and $\Delta \Omega$ can be determined. For instance, for the semi-annual averages of the active longitude shifts shown in Fig. 1, we determine mean, area weighted spot latitudes $\psi_{i}$ for $\Delta T=0.5$ year from actual sunspot data. The best fit to the migration paths are shown by the tracks in Fig. 1. The best fit parameters together with Eq. (1) define a new, dynamic reference frame in which the active longitudes persist at the same location. The panels for the Northern and Southern hemispheres show primary and secondary maxima in the longitudinal sunspot distribution. The maxima follow two trajectories, visible in each panel. Usually one of the two active longitudes is more active than the other. Then a flipflop occurs, and the active longitudes exchange dominance.

We defined the expected migration path of active longitudes, obtained using Eqs. (1) and (2), as the new dynamic reference frame, in which the active longitudes are stable. The semi-annually averaged active longitudes in this dynamic reference frame are shown in Fig. 2. The corrected longitudes of spot concentrations occupy two wide bands separated by about $180^{\circ}$, arguing for the persistence of the active longitudes. The distribution of the corrected active longitudes (Fig. 2) is significantly more regular and apparent than that presented in Fig. 1, especially for the primary spot. This plot is made mostly
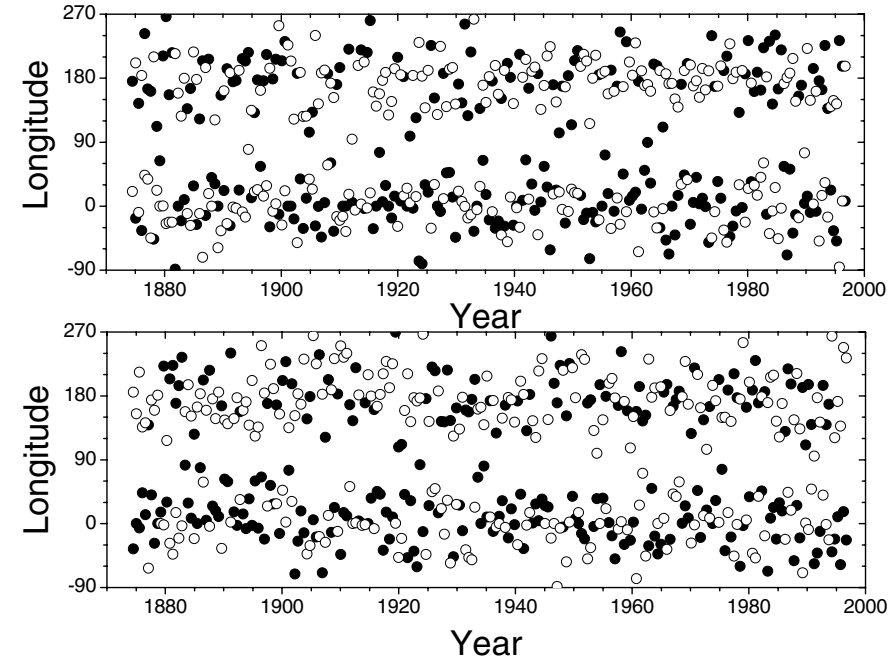

Fig. 2. The semi-annually averaged active longitudes for the Northern (upper panel) and Southern (lower panel) hemispheres in the dynamic reference frame defined by Eqs. (1) and (2), i.e. after the migration paths in Fig. 1 have been subtracted from the data points. Filled and open circles represent the dominant and secondary active longitudes respectively. Note that, because of the flip-flop phenomenon, the two active longitudes are prominent even in the distribution of the single dominant (or secondary) active region.

for the purpose of visualization of the phenomenon of active longitudes, as an appropriate averaging emphasizes the visibility of the phenomenon.

In the next step we applied the same correction procedure not to the spot concentration longitudes but to the raw 

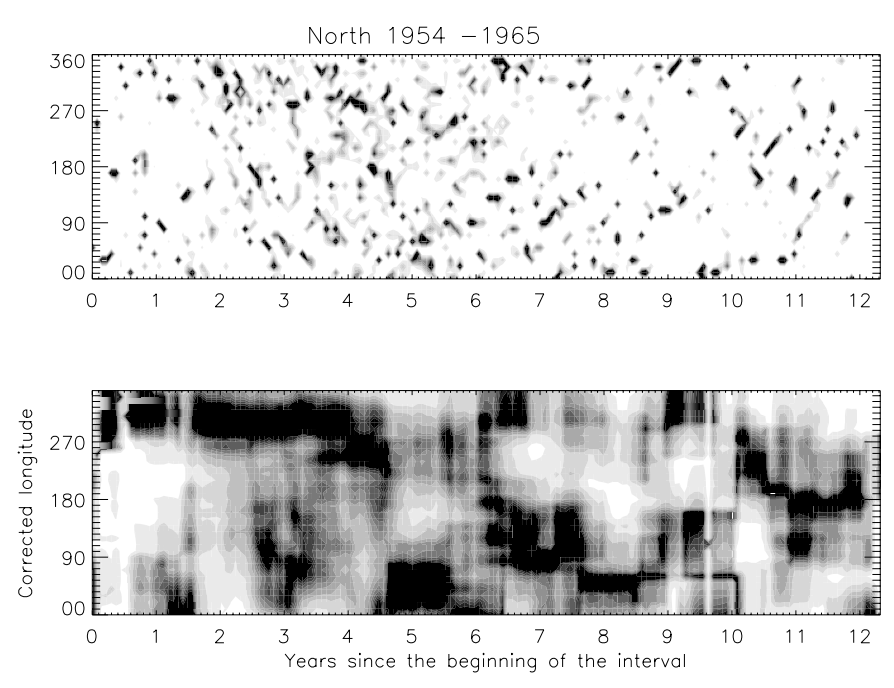

Fig. 3. Corrected longitudes of the normalised sunspot occurrence in the Northern hemisphere during the solar cycle N 19 reduced to the dynamic reference frame (upper panel). We show the same plot smoothed by the Lee filter (Lee 1986) in the lower panel.

individual sunspot data. A sample of the area-weighted sunspot distribution in the corrected longitude system is shown in Fig. 3 (upper panel) for the Northern hemisphere for cycle No. 19 (1954-1965). The sunspot area distribution yields two similar bands, $180^{\circ}$ apart, but this is not clearly distinguishable. To reduce the noise, we have smoothed the plot using the Lee filter which smooths image noise by generating statistics in a local neighbourhood and comparing them to the expected values (Lee 1986). The result of this smoothing is presented in the lower panel of Fig. 3. Note that this filtering technique smooths only the image of the upper panel but not the sunspot data. It is important that the active longitudes can be also found in the raw sunspot data, without any pre-processing or smoothing of the actual sunspot data (see Usoskin et al. 2005). This confirms that the detection of active longitudes is real, contrary to the doubts expressed by Pelt et al. (2005), and that the nonaxisymmetric magnetic structures underlying these active longitudes are subject to a differential rotation.

A dynamic reference system with the above definition was introduced by Usoskin et al. (2005) using all observed sunspots, without averaging, over the interval $\Delta T$ of one Carrington rotation. For a period covering 120 years (1874-1996) they found the best fit parameters of the differential rotation (Eq. (2)) to be: sidereal $\Omega_{0}=14.33 \pm 0.01$ $(14.31 \pm 0.01)$ and $\Delta \Omega=3.40 \pm 0.03(3.39 \pm 0.02) \mathrm{deg} / \mathrm{day}$ for the Northern (Southern) hemispheres, respectively. When compared with the rotation law determined by Schou et al. (1998) from surface Doppler shifts observed with SOHO/MDI, active longitudes seem to rotate faster than the surface plasma at lower latitudes (within the belt of $\pm 20^{\circ}$ ) and with a similar rate or slower at higher latitudes. On the other hand, the rotation rate of the active longitudes is also close to that determined from helioseismological data (Schou et al. 1998) for the region around fractional radius 0.75 , which is deeper than that associated with the rotation of individual sunspots. This might be

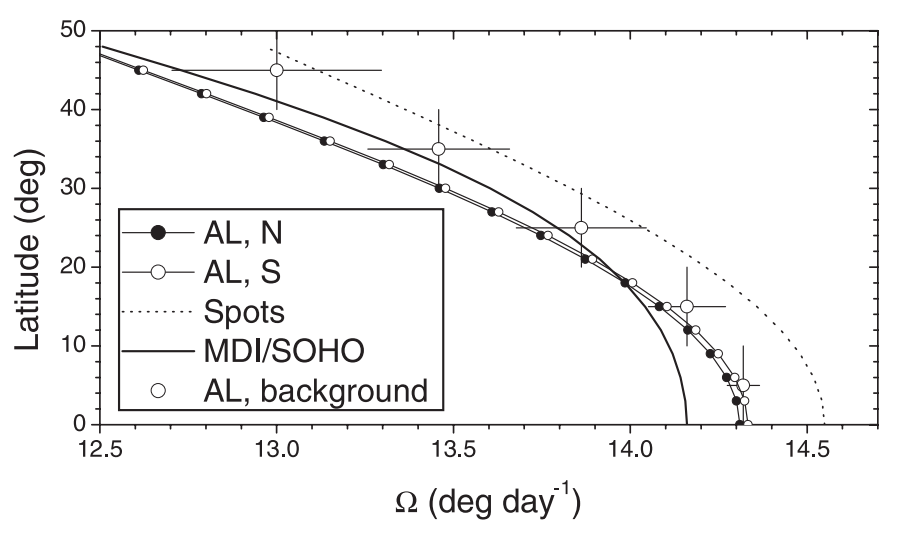

Fig. 4. The sidereal differential rotation as defined for the active longitudes in the Northern and Southern hemispheres separately (Usoskin et al. 2005), in comparison with that using different tracers: surface Doppler shifts by SOHO/MDI (Schou et al. 1998) and individual sunspots (Balthasar et al. 1986). Big dots with error bars correspond to dominating rotation periods defined from active longitudes of the background magnetic field in different latitudinal belts by Bobova \& Stepanian (1994) for the period 1969-1980.

connected with the fact that various tracers of solar rotation can be associated with different depths in the solar interior.

Indeed, the parameters of the differential rotation may vary significantly when estimated by means of different tracers (Fig. 4). For instance, sunspots rotate faster than the ambient surface plasma (Balthasar et al. 1986; Schou et al. 1998). Also, the angular velocity as estimated from the sunspot-based active longitudes differs significantly from that for individual spots. At first appearance, this comparison seems to imply that the active longitudes are not linked to the depth of individual sunspot anchoring (see however Sect. 3).

Differential rotation of active longitudes has also been recognized by earlier studies. Bumba \& Howard (1969) found long-lived sections of alternating polarity in old and new magnetic flux within a narrow longitudinal zone and different latitudinal zones, from observations in 1959-1967. They noticed that the structures associated with strong magnetic fields seemed to resist the shearing effect of the differential rotation. Bobova \& Stepanian (1994) analyzed the dominant periodicities of the low-intensity surface background solar magnetic field in the range around 13.5 and 27 days (related to active longitudes) and their dependence on the solar latitude for the period 1969-1980. In agreement with the earlier study of Bumba \& Howard (1969) they found that the active longitudes in the background magnetic field rotate differentially (see their Fig. 2), and this differential rotation law (dots in Fig. 4) is close to that discussed above. Bumba (1991) also found active longitudes to have different rotation rates at different latitudes, and Bai (2003) found the same phenomenon in the distribution of solar flares. Benevolenskaya et al. (1999b) determined rotation rates of old and new magnetic flux within an active longitude, observed during the transition period from solar cycle 22 to 23 and compared them with the internal rotation inferred by helioseismology. They suggested that both fluxes were probably generated in a low-latitude zone near the base of the 
solar convection zone. All these results support the conclusion of Berdyugina \& Usoskin (2003) and Usoskin et al. (2005).

The study by Usoskin et al. (2005) has made it possible to quantify the observed level of the nonaxisymmetry in the sunspot distribution in the following way. Let $\lambda_{1}$ and $\lambda_{2}=$ $\lambda_{1}+180^{\circ}$ be the mean active longitudes in the dynamic reference frame (see e.g. Fig. 2). Then each spot with reduced longitude $\lambda$ is considered as belonging to the nonaxisymmetric structure, provided that $\left|\lambda-\lambda_{1}\right|$ or $\left|\lambda-\lambda_{2}\right|$ does not exceed $45^{\circ}$ (longitude is circular). Let $N_{1}$ be the area of sunspots in the vicinity of the proposed active longitudes, thus containing contributions from both the axisymmetric and nonaxisymmetric contributions, and let $N_{2}$ be the area of the remaining sunspots which do not fall into these $\pm 45^{\circ}$ bands around the active longitudes (and so considered to belong to the axisymmetric structure). Then the measure of nonaxisymmetry is defined as

$\Gamma=\frac{N_{1}-N_{2}}{N_{1}+N_{2}}$

$\Gamma$ can take values between 0 and 1 , so that $\Gamma=0$ corresponds to a longitudinally uniform distribution of spots $\left(N_{1}=N_{2}\right.$, i.e. an axisymmetric distribution), and $\Gamma=1$ implies that all spots appear around the active longitudes. This value of $\Gamma$ for the sunspot area distribution appears to be quite modest, being about 0.1 for the entire time interval since 1874 , and does not exceed 0.25 for individual cycles. Although the nonaxisymmetry is rather weak, its statistical significance is very high. The nonaxisymmetry of 0.1 implies that the ratio between nonaxiand axisymmetric distributions is about 1:10. We note that the nonaxisymmetric structures become less pronounced when the above analysis is applied to the data without separating the Northern and Southern hemispheres; this is a consequence of the observed lack of synchrony between the hemispheres.

\section{Active longitudes and nonaxisymmetric magnetic configurations}

We can summarize the following features related to the existence of solar active longitudes which need to be taken into account and reproduced by an appropriate dynamo model.

- There are two active longitudes approximately $180^{\circ}$ apart, that can persist, maintaining phase, over at least 10 solar cycles.

- The active longitudes are affected by differential rotation and migrate in Carrington longitude according to the mean latitude of sunspot formation/manifestation.

- The nonaxisymmetry contribution is quite small, at about $1 / 10$ of that from the dominant axisymmetric mode.

- The differential rotation responsible for the migration of active longitudes is different from that obtained from individual spots as tracers, which seems to imply that these phenomena are anchored at different depths.

- At any given time, one of the two active longitudes is more active (dominant), and the dominance switches from time to time between these two active longitudes: this is known as the "flip-flop" phenomenon.
Assuming that the above description of the active longitude phenomenon is associated with some sort of large-scale magnetic structure, we now seek to provide a consistent theoretical explanation. An important point is that the sunspot data represent only the behaviour of the strongest part of the solar activity wave and give no direct information concerning the form of the magnetic field throughout the entire convective zone. We know from the sunspot data (as a function of time $t$ ) the migration law at the latitude $\psi(t)$ and longitude $\lambda(t)$ where the activity wave is strongest at time $t$. We assume the migration law to be axisymmetric. However, the sunspot data give us no information about the position of the strongest toroidal magnetic field at each instant $t$ at every latitude $\psi$. In contrast, a theoretical interpretation of the observational data depends strongly on such behaviour. Suppose that the centre of the migration wave is at latitude $\psi(t)$ at time $t$ - this is the latitude that the active longitude analysis experiences. Thus, if $\lambda^{*}(\psi, t)$ is the longitude at which the magnetic field is maximal at latitude $\psi$ at time $t$, then $\lambda(t)=\lambda^{*}(\psi(t), t)$ is the derived active longitude. It is important to note that we cannot completely reconstruct $\lambda^{*}(\psi, t)$ from $\lambda(t)$ and $\psi(t)$ alone.

A MHD-interpretation of the phenomenon of active longitudes requires some hypothesis concerning the general shape of the function $\lambda^{*}(\psi, t)$. It can be assumed that the rotation rate $\omega$ of the longitude $\lambda^{*}$ at various latitudes is specific, i.e. $\partial \lambda^{*} / \partial t=\omega(\psi)$ and $\omega\left(\psi_{1}\right) \neq \omega\left(\psi_{2}\right)$. Then we identify $\omega(\psi)$ with the rotation rate $\Omega$, as Eq. (2). The magnetic structure underlying the active longitude in the dynamical reference frame has a unique longitudinal maximum for all latitudes. This is the most straightforward understanding of the observational data. We shall refer to it below as the concept of true differential rotation of active longitude. It is important to distinguish the ongoing winding of structures by the rotation, as occurs here, from a fixed degree of twisting.

An alternative interpretation is that the nonaxisymmetric magnetic structure underlying the active longitudes is strongly affected by differential rotation, while the rotation law for the magnetic structure is still solid body. This explanation of the differential rotation of active longitudes is based on a stroboscopic effect. The region of enhanced magnetic field migrates from the middle latitudes to the solar equator along the line $\lambda^{*}(\psi, t)$. Because this line is affected by differential rotation, it gives the impression of a differential rotation of active longitude. In this case, one should make a distinction between $\omega$ which is here $\psi$-independent and $\Omega$ from Eq. (2) which depends on $\psi$. The latter dependence reflects the curvature of the nonaxisymmetric structure as well as latitudinal migration of the region of enhanced magnetic field. We refer below to this concept as the stroboscopic effect.

We illustrate the action of the stroboscopic effect by the following example. We take the mean latitude of sunspot distribution at a given instant from the Greenwich Royal Observatory data - this is the same data as used by Berdyugina \& Usoskin (2003), Usoskin et al. (2005) - see the upper panel of Fig. 5. Then the angular velocity at the same latitude is found from Eq. (2) and the longitudinal lag from integrating Eq. (1). The result presented in the lower panel of Fig. 5 is remarkably similar to the plot in the left hand panel of Fig. 1. 

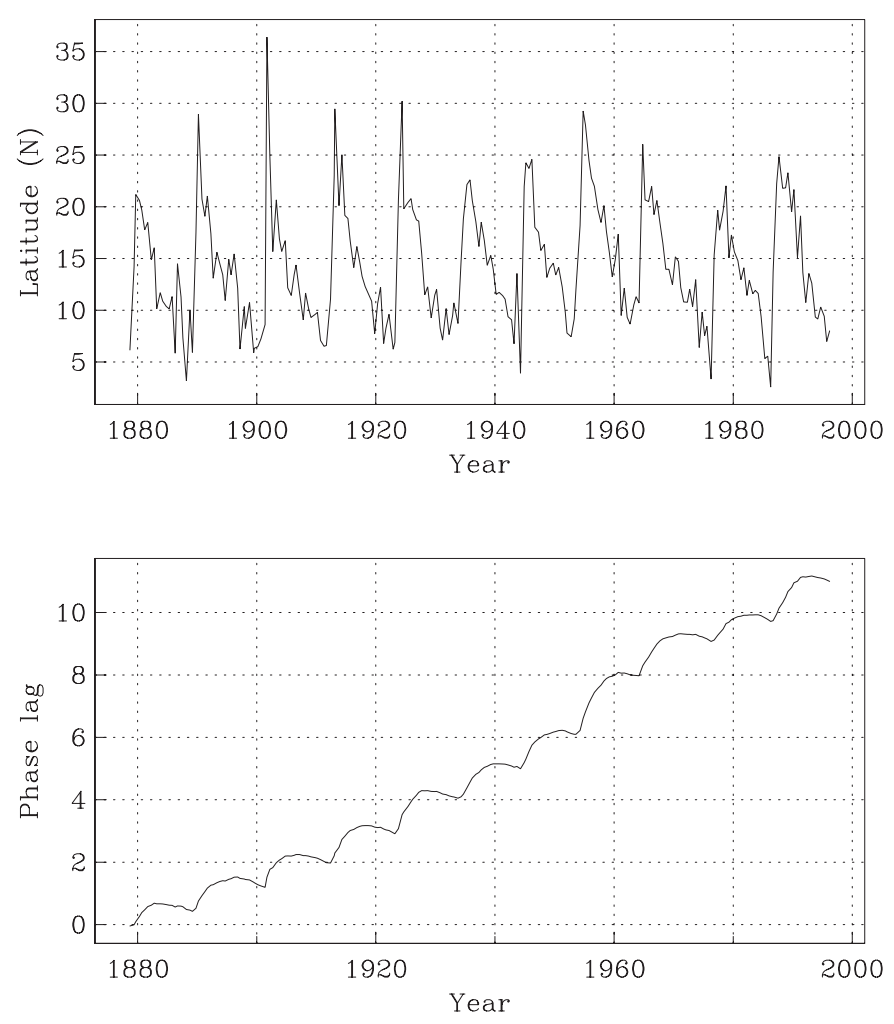

Fig. 5. The stroboscopic effect illustrated. The upper panel shows the mean latitudes of the sunspot distribution for the Northern hemisphere as a function of time, and the lower panel gives the corresponding phase lags $\Delta \Lambda / 360^{\circ}$, computed assuming a stroboscopic effect, as described in Sect. 2 to be operative.

The sunspot data are inadequate for distinguishing between true differential rotation of active longitudes and the stroboscopic effect. The point is that the sunspots near the centre of the instantaneous locus of maximal density of the distribution have peculiar motions, which can in principle mimic a particular behaviour of $\lambda^{*}$. Data from additional tracers of solar activity are also needed to resolve this controversy. We note however that the sunspot data do give some hints supporting the stroboscopic interpretation. In particular, in the framework of the concept of true differential rotation it is necessary that the rotation law of the nonaxisymmetric part of the solar magnetic field differs from that of sunspots taken as an entirety, whilst both rotation rates are reconstructed from the same body of sunspot data. The other point is that, in the case of true differential rotation, it would be more natural to have a curve that diverges more and more from the general trend in Fig. 1 as time increases, rather than more or less stable oscillations.

Explanations involving true differential rotation appear to be much more severely constrained in the context of any MHD-based theory than ideas connected with a stroboscopic effect.

\section{Active longitudes in the light of theory}

Now we consider various theoretical approaches to solar magnetic fields from the viewpoint of their ability to reproduce the phenomenology of active longitudes discussed above.

\subsection{Mean-field dynamos and active longitudes}

Starting from the famous paper of Parker (1955), conventional dynamo models for the large-scale solar magnetic field are mainly axisymmetric. The occurrence of active longitudes as discussed above appears unexpected in this context. However the degree of incompatibility with the basic ideas of solar dynamo theory seems to be slightly exaggerated. First of all, the conventional solar dynamo modes describe the mean magnetic field, i.e. the magnetic field averaged over substantial temporal or spatial scales or over a statistical ensemble of convective fluctuations, while the sunspot data do not include such averaging, at least not in an explicit form. There is no doubt that the total magnetic field distribution inside the Sun is asymmetric (from Cowling's theorem, if common sense alone is not sufficient!). Strictly speaking, the discrete nature of sunspot data is sufficient for some deviation from axial symmetry.

The discrepancy between mean and total magnetic field distribution could simply be manifested as active longitudes in the observational data. However it is hardly to be expected that this would appear in the form of two long-term preferred longitudes, as visible in Figs. 1 and 2. A set of chaotic patterns might be expected, rather than the coherent structures seen in these figures. The situation is, however, not so straightforward. Consider for example the geomagnetic field. The geomagnetic field is not completely axisymmetric, and because the Earth's magnetic and geographic poles do not coincide, two "active" geographical longitudes are defined. This asymmetry is to some extent similar to the asymmetry of the solar magnetic field. The geodynamo and the solar dynamo obviously differ, but nevertheless the basic physics of these processes is similar. It is possible to consider the displacement between the Earth's magnetic and geographic poles as a fluctuation that can be removed after temporal averaging. This possibility looks plausible enough to merit a more detailed investigation. The Earth's magnetic poles seem much less stable - their displacement is significant on the timescale of a century - to be compared with the geodynamo timescale of about $10^{5}$ years, whereas the persistence of the solar active longitudes should be compared with the time-scale of solar dynamo (activity cycle length). Thus we do not consider a priori this explanation to be promising in the solar context.

In principle, the active longitudes could be considered as representing domains where sunspot formation is somehow easier than elsewhere on the solar surface. For example, after an active region is created by whatever mechanism, formation of the next region nearby could, in principle, be easier than before (e.g. Ruzmaikin 1998). Such an scenario would have the same problems as discussed above, in terms of coherence and long-term stability.

\subsection{Nonaxisymmetric dynamo modes}

Nonaxisymmetric magnetic structures are unusual but possible in mean-field spherical dynamo models. A quantitive analysis of this aspect of the kinematic dynamo problem has been recently undertaken by Bassom et al. (2005) in terms of the Parker migratory dynamo. We present here the main 
conclusions of this rather mathematical paper. The basic mechanism of the solar dynamo as suggested by Parker (1955) can be described as follows. The mean magnetic field is considered as a combination of toroidal $B_{\mathrm{T}}$ and poloidal $B_{\mathrm{P}}$ fields. Due to radial gradients of the angular velocity, a toroidal field is produced from the poloidal $\left(B_{\mathrm{P}} \rightarrow B_{\mathrm{T}}\right)$. To close the chain of magnetic field self-excitation, it is necessary to produce poloidal magnetic field from toroidal $\left(B_{\mathrm{T}} \rightarrow B_{\mathrm{P}}\right)$. The helicity of the convective motions in a rotating body provides such an mechanism, via the $\alpha$-effect. The joint action of the differential rotation and the $\alpha$-effect has to be strong enough to overcame the destructive role of turbulent diffusivity. This scheme, known as the $\alpha \omega$-dynamo, obviously discriminates against nonaxisymmetric magnetic structures. The point is that any kind of nonaxisymmetry increases diffusive losses of magnetic energy. Radial shear in the angular velocity winds up the nonaxisymmetric field, bringing oppositely directed field lines closer together (e.g. Rädler 1986). For a solar-like rotation curve the latitudinal dependence of the angular velocity is quite pronounced. Consider two nearby latitudinal belts with angular velocities $\Omega(\theta)$ and $\Omega(\theta+\Delta \theta)$ - we introduce now the polar angle $\theta$ for use in these more theoretical sections, with $\theta=0,180^{\circ}$ corresponding to North and South poles respectively. The relative rotation rate of the belts is $(\partial \Omega / \Omega \partial \theta) \Delta \theta$. This relative rotation does not affect an axisymmetric magnetic configuration, but stretches the nonaxisymmetric field. When this stretching leads to a differential rotation of the nonaxisymmetric magnetic field, rather than a constant offset of nonaxisymmetric field features with latitude, this is known as phase mixing. The phase mixing also enhances the losses caused by turbulent diffusion. The leading dynamo eigenmode is normally axisymmetric and its growth rate is determined by the maximal value of $\alpha \partial \Omega / \partial r$. For a nonaxisymmetric eigenmode, the dynamo has to overcome the effect of phase mixing, and growth rates appear to be lower than for axisymmetric eigenmodes.

The situation becomes more delicate, when the ability of the $\alpha$-effect to produce toroidal magnetic field from poloidal is also taken into account, in the framework of the so-called $\alpha^{2} \omega$-dynamo (e.g. Roberts \& Stix 1972). Of course, the role of the $\alpha$-effect in the link between $B_{\mathrm{P}}$ and $B_{\mathrm{T}}$ is usually less important than that of differential rotation (the opposite limit is referred to as the $\alpha^{2}$-dynamo). The two generators of toroidal field, i.e. differential rotation and helicity, do not necessarily harmonize their effects. There is a parametric domain, where a nonaxisymmetric magnetic structure can become preferred, in spite of the losses caused by enhanced turbulent diffusion. The reason for this preference is that the domain of field generation is shifted (in physical space) from a point where the value $\partial \Omega / \partial r \Delta \theta$ is maximum to some nearby point where the joint efforts of differential rotation and helicity in reproducing $B_{\mathrm{T}}$ from $B_{\mathrm{P}}$ are more concerted.

We stress however that a nonaxisymmetric kinematic dynamo mode, even if preferably excited, has a solid-body rotation without exhibiting any effect of phase mixing. In other words, a linear dynamo instability excites a magnetic structure growing and preserving its form. This structure is twisted by differential rotation but is not being progressively wound up. The stroboscopic effect is thus possible. These statements generally apply also to nonlinear dynamo solutions. This sort of mechanism appears compatible with the stroboscopic concept but not with the concept of true differential rotation of active longitudes. In particular, the absence of phase mixing means that a true differential rotation of active longitudes is impossible. Note, that the discussion of Bassom et al. (2005) takes into account only true differential rotation.

Nonaxisymmetric magnetic structures excited by the kinematic $\alpha^{2} \omega$-dynamo have low azimuthal wave numbers, $\boldsymbol{B} \sim$ $\operatorname{expim\phi }$ with $m=1$ usually ( $m=2$ appears as a very rare exception). Fluri \& Berdyugina (2005) suggested that a combination of $m=1$ and $m=2$ modes might explain some active longitude and flip-flop cycles. Such a combination might be useful to explain active longitudes that jump by either $90^{\circ}$ or $180^{\circ}$. Moss $(2004,2005)$ discussed the possibility of active longitudes separated by less than $180^{\circ}$, as well as possible mechanisms for their excitation (although not by excitation of a $m=2$ mode); Korhonen \& Elstner (2005) claim that such active longitudes are visible on FK Com.

Nonaxisymmetric magnetic structures generated by a dynamo in its kinematic stage can survive during the nonlinear stage of the dynamo evolution. Nonlinear stellar dynamo models with stable large-scale nonaxisymmetric fields were obtained many years ago. The earliest results here are Rädler et al. (1990) and Moss et al. (1991a), although these have rather artificial rotation laws, of the form $\Omega=\Omega(r)$. More recently, Moss (1999) studied a solar model with a rotation law derived from helioseismological observations, and found that a weak nonaxisymmetric field could coexist with a dominant axisymmetric field. Moss $(2004,2005)$ and references therein discuss some recent developments in this area. Nonlinear dynamo models of the latter kind can in principle provide stable nonaxisymmetric magnetic structures which demonstrate a flip-flop phenomenon.

We conclude that the solar dynamo could in principle generate nonaxisymmetric magnetic configurations, comparable in many respects with the active longitudes phenomenon as recorded in sunspot and other data. Again, the dynamo excited configurations do not demonstrate phase mixing and are compatible only with a stroboscopic interpretation.

One way in which this difficulty might be avoided, and at least a degree of phase mixing introduced, can be envisaged as follows. There is a quite uncertain relation between the generation of global-scale magnetic field by a mean field dynamo, and the formation of sunspots - similar considerations apply to both axisymmetric and nonaxisymmetric fields. If the sunspots are born as a result of some kind of instability of the large-scale field, around depth $d$ say, and effectively become detached from the large-scale field, then they will be transported according to the angular velocity of their birth latitude at depth $\lesssim d$. Dynamo models for the solar cycle all implicitly assume some such link between sunspots and the axisymmetric large-scale field: the relatively weak nonaxisymmetric field can be assumed to add a small bias to the formation process. Thus, as the axisymmetric field migrates equatorwards, sunspots near the active longitudes will experience a different local rotation rate, i.e. phase mixing. However, in the absence of any stroboscopic effect, 
there is still the question as to why these sunspots arise from a different depth than the generality of spots.

The properties of dynamo models become richer if any underlying nonaxisymmetric hydrodynamics are considered. Although this is a natural consideration for close binaries (Moss et al. 2002), nevertheless it is not a priori excluded that, say, the meridional circulation in the solar interior could deviate from axisymmetry. Barker \& Moss (1994) and Moss et al. (1995) considered active longitude excitation that is promoted to some extent by meridional circulation. As far as it is known however nonaxisymmetric dynamo models with nonaxisymmetric flows do not directly demonstrate phase mixing.

Note that models in which dynamo action occurs in spatially distinct regions, e.g. a combination of tachocline and surface dynamos (e.g. Mason et al. 2002) might expand the range of dynamo explanations for active longitudes. Brandenburg (2005) has recently discussed the possibility that the solar dynamo is largely controlled by the near-surface shear layer, where the angular velocity approaches each of the values discussed within quite a small radial extent. However it is unclear how such a mechanism would generate or interact with a largescale nonaxisymmetric field.

\subsection{Beyond a dynamo explanation}

The dynamo explanations outlined above for nonaxisymmetric structures are limited to a particular range of choices of the dynamo governing parameters. It is far from obvious that these are compatible with solar hydrodynamics. A reasonable next step is to seek an explanation based on a field which is essentially passive in the convective zone.

Let us consider a relic magnetic field frozen into the solar radiative zone sometime during the early stages of solar evolution, and surviving there until now. The conductivity of the solar interior is high enough and, according to the helioseismological data, the rotation shear in the radiative core is small enough to allow in principle the existence of such a relic field. Indeed, the presence of even a relatively weak $(\gtrsim 1 \mathrm{G}$, see e.g. Mestel 1961; Mestel \& Weiss 1987) field could promote a state of uniform rotation in the core. The concept of a relic magnetic field hidden within the solar radiative zone, as well as its possible manifestation in the form of an nonaxisymmetric magnetic field configuration has been discussed a number of times (e.g. Dicke 1979; Bravo \& Stewart 1996; Kitchatinov et al. 2001; Mordvinov \& Kitchatinov 2004). For example, Kitchatinov et al. (2001) suggest that a relic field is captured, producing a nonaxisymmetric structure with strength somewhat below $1 \mathrm{G}$. Taking into account the discrepancy between the amplitudes of toroidal and poloidal magnetic field at the solar surface and the stretching of the toroidal field in the tachocline region, manifestation of an active longitude phenomenon in a form comparable with observations is possible. Because the captured magnetic field is taken as frozen into the radiative core but subject passively to the differential rotation of the envelope, a phase mixing of active longitudes that results directly from this relic field cannot be expected. But, just as in Sect. 4.2, if the effect of the nonaxisymmetric field is simply to bias slightly the probability formation from the dominant axisymmetric field, then phase mixing is possible. However, another difficulty now arises: the rotation rate of the active longitudes should now be that of the core, where the relic field is anchored. This is not observed.

In principle, it might be believed that the active longitudes as known from sunspot data are mainly associated with the sunspot formation mechanism rather than reflecting directly a dynamo generated or relic nonaxisymmetric magnetic structure. Such viewpoint appears favourable for the true differential rotation of active longitudes, but problems concerning sunspot formation remain far beyond the scope of this paper.

The above estimate for the strength of relic nonaxisymmetric structures is very modest. For example, Goode \& Thompson (1992) claim that a relic field strength up to $30 \mathrm{MG}$ (!) would not contradict the helioseismological data, although its consequences for solar dynamo theory might be more marked (Pudovkin \& Benevolenskaya 1982; Boyer \& Levy 1984). Here we avoid any discussion concerning the possible strength of the solar relic magnetic field, attempting to estimate to what extent a relic field present in the radiative core might penetrate into the convection zone, and subsequently be manifested at the solar surface.

In order to examine possible effects of the relic field, we carried out the following numerical experiment. We consider a solar-like rotation law, and impose the presence of a nonaxisymmetric relic field of even symmetry with respect to the rotational equator ("perpendicular dipole-like") as an inner boundary condition on a mean field dynamo calculation, using essentially the nonlinear nonaxisymmetric dynamo code described in Moss et al. (1991a). We modelled this by taking the given rotation law in the region $r>r_{\mathrm{b}}$ (see Fig. 1 of Moss \& Brooke 2000), with $r_{\mathrm{b}}=0.64 R$ and the region $r_{\mathrm{b}}<r \leq 0.70 R$ being a transition region between the rigidly rotating "core" region $r \leq r_{\mathrm{b}}$ and the "convection zone" $r>0.70 R$. To ensure smoothness of the numerical solution, we actually impose the field by a boundary condition at radius $r=0.58$, with a region of uniform rotation and finite diffusivity in $0.58<r \leq 0.64$.

As might be expected, differential rotation tends to wind up and destroy the nonaxisymmetric field. Thus we examined a very favourable case with almost the smallest value of the standard dynamo parameter $C_{\omega}=\Omega_{0} R^{2} / \eta$ that gives oscillatory axisymmetric dynamo solutions $\left(\Omega_{0}\right.$ is here the core angular velocity). We show in Fig. 6 the dependence of the radial component of the $m=1$ field on radius, at a latitude of about $32^{\circ}$. This corresponds to a dipole field strength of 0.01 at radius 0.58 (units of $\boldsymbol{B}$ are equipartition field strength).

We see that the nonaxisymmetric field strength at the surface is about $1.5 \%$ that at $r=0.58$. The contours of surface radial field are shown in the upper panel of Fig. 7, where the nonaxisymmetric component is barely discernable.

When interpreting this experiment it is necessary to remember that

(i) the dynamo parameters used are optimal for survival/penetration of nonaxisymmetric field, and so very probably underestimate the effects of differential rotation; 


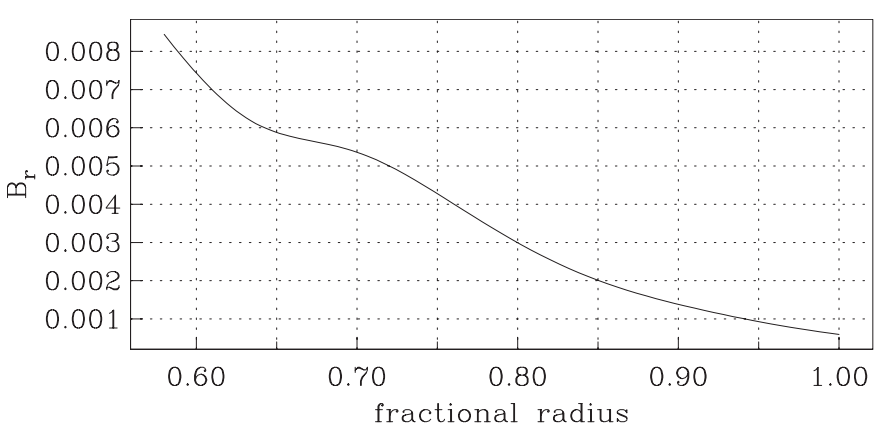

Fig. 6. The dependence of the radial component of nonaxisymmetric imposed (relic) field on radius. The ratio of the strength at the surface to that imposed at the core is about 0.09 .
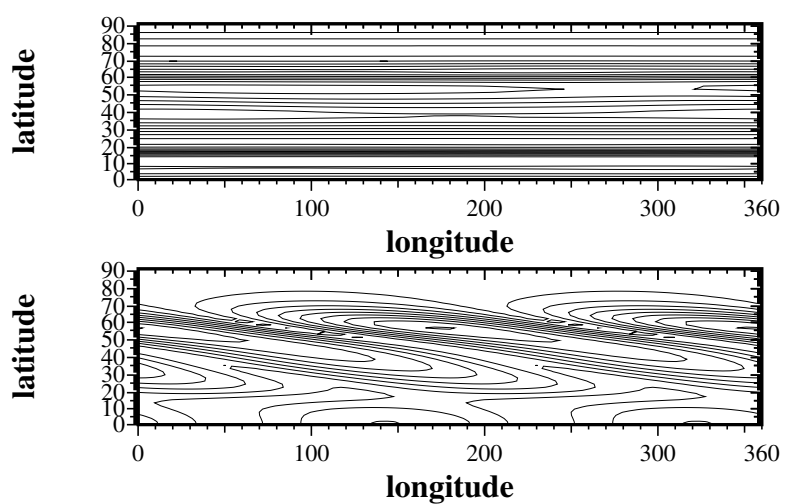

Fig. 7. The upper panel shows isocontours of the total magnetic field strength at fractional radius $r=0.81$ in the Northern hemisphere. The lower panel shows isocontours of the strength of the nonaxisymmetric part of the field in the same hemisphere. In this illustrative computation, the field strengths are symmetrical about the rotational equator. The deviations of the total field strength from axisymmetry are very small. The nonaxisymmetric component shows the shearing effect of the latitudinal differential rotation.

(ii) the exact depths from which the visible manifestations of active longitudes arise is not known, however, it seems to be deeper than that for anchoring of sunspots.

However, although a relic perpendicular dipole-like field could in principle underly persistent active longitudes separated by $180^{\circ}$, it is difficult to see how it could be connected directly with any alternation of longitudes, although a "displaced dipole" could certainly make one longitude permanently more active than the other. Rather, as hinted at by Fig. 7 (lower panel), the nonaxisymmetric structure will be sheared into an arc-like pattern, centred on the equator. Figure 7 shows that additional modulation of the field structure by the differential rotation can also occur. The fundamental difficulty with this type of explanation appears to lie with the rotation rate of the active longitudes, which is necessarily that of the core region.

We conclude that the relic field idea encounters significant problems in explaining the active longitude phenomenon.

\subsection{Nonlinear instabilities}

A less conventional explanation for the active longitudes might be found in various nonlinear instabilities based on a

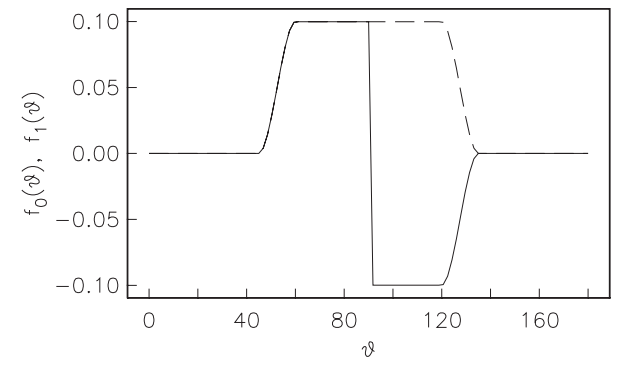

Fig. 8. The functions $\delta \alpha_{0} f_{0}(\theta)$ (broken), $\delta \alpha_{1} f_{1}(\theta)$ (solid) that determine the latitudinal dependence of the oscillating $\alpha$-perturbation (Sect. 4.4). $\theta$ is the polar angle. In $\theta<90^{\circ}$, the graphs coincide.

nonlinear interaction between the magnetic field and hydrodynamical flows. We can mention here an instability discussed by Dikpati et al. (2004). The instability arises from kinetic and magnetic drag forces in the solar tachocline. As a result, the conventional toroidal component of the solar dynamo wave, in the form of two latitudinal strips of enhanced magnetic field propagating equatorialwards from middle latitudes to the solar equator, changes its form near the equator. They become inclined to the solar equator, resulting in two preferred longitudes, i.e. the longitudes of the lowest and highest latitudes of the strip. The instability looks attractive when considering the problem of active longitudes, because it does not require special tuning of the dynamo governing parameters, and gives only a modest level of nonaxisymmetry. It also introduces a breaking of equatorial symmetry, which may be helpful given that deviations from dipole symmetry are observed in solar magnetic phenomena (of course, it is possible for such symmetry breaking to occur spontaneously). The latitudinal displacement of toroidal strips can be different in the Northern and Southern hemispheres. This could explain why the active longitudes become more pronounced when the data for the Northern and Southern hemisphere are treated separately. The flip-flop phenomenon could be attributed, say, to the instant at which the lower latitude of the propagating toroidal strip reaches the equator. An obvious advantage of this explanation is that it provides the possibility presenting the problem of active longitudes in the form of the inclination of the solar magnetic equator (Bravo \& Stewart 1996).

We attempted to model this possibility in a very preliminary manner by introducing a perturbation to the alpha-coefficient, with $m=0$ and $m=1$ parts, the latter corotating with the equator at fractional radius $r_{\mathrm{c}}$, and the perturbation oscillating with frequency $\omega_{\alpha}$. Thus

$\delta \alpha=\alpha_{0}(r, \theta)\left(\delta \alpha_{0} f_{0}(\theta)+\delta \alpha_{1} f_{1}(\theta) \cos \left(\phi-\Omega_{\mathrm{c}} \tau\right)\right) g(r) \cos \omega_{\alpha} t,(4)$

where $f_{0}(\theta)$ and $f_{1}(\theta)$ are respectively antisymmetric and symmetric about the equator (see Fig. 8), $g(r)=[\cosh (2(r-$ $0.7) / 3)]^{-2}, \delta \alpha_{0}=\delta \alpha_{1}=0.1, \Omega_{\mathrm{c}}=\Omega\left(r_{\mathrm{c}}, \pi / 2\right) . \alpha_{0}(r, \theta)$ is the unperturbed alpha-term. Thus the perturbation can be considered to be localized near the equator at the bottom of the convection zone, to rotate with angular velocity $\Omega_{\mathrm{c}}$, and to oscillate about the equator with amplitude $\delta \alpha_{0}+\delta \alpha_{1}=0.2$.

We show in Fig. 9 the evolution with time of the parities and global energies of the $m=0$ and $m=1$ parts of the field 

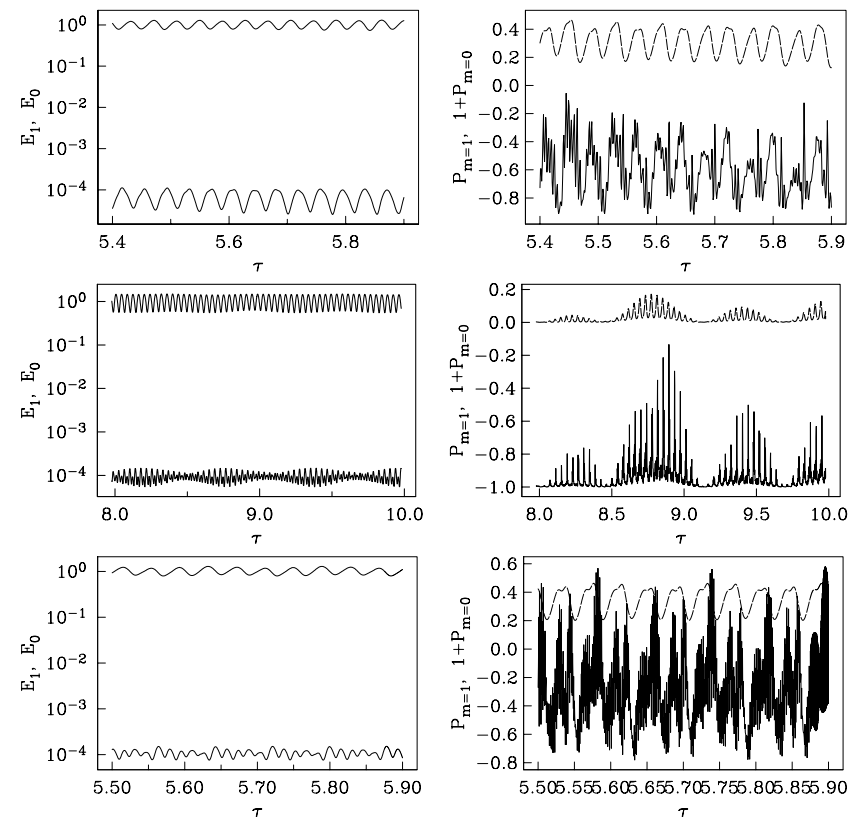

Fig. 9. Left hand panels: times series of energies $E_{0}, E_{1}$ in the axisymmetric and nonaxisymmetric parts of the field (respectively the upper and lower curves in each panel). Right hand panels: times series of the global parities $P_{0}, P_{1}$ of the axisymmetric and nonaxisymmetric parts of the field (respectively the upper and lower curves in each panel). Note that $P_{0}+1$ is plotted, to avoid confusion between the curves. From top to bottom: $\omega_{\alpha}=(1,2,2.5) \times \Omega_{\text {cyc }}-$ see Sect. 4.4.

for cases with $\omega_{\alpha} \approx(1,2,2.5) \times \Omega_{\text {cyc }}$ respectively, where $\Omega_{\text {cyc }}$ is the oscillation frequency of the axisymmetric part of the magnetic field when $\delta \alpha=0$. At the surface the nonaxisymmetric field strength is at most a few percent of that of the axisymmetric field. The unperturbed solution $(\delta \alpha=0)$ has a stable state with $P_{0}=-1$. Unsurprisingly, the introduction of a disturbance lacking equatorial symmetry introduces deviations from a pure parity state. Nevertheless, the dependence of the form of the variations in energy and parity on the value of $\omega_{\alpha}$ (Fig. 9) is striking. It might be tempting to associate such fluctuations in parity with the known deviations of the solar field from strict equatorial antisymmetry (e.g. Pulkkinen et al. 1999; Knaack et al. 2004). Whilst noting that these solutions inevitably posses certain "preferred" longitudes, we have not attempted to investigate whether they exhibit unambiguous flip-flop behaviour.

Recently, Bigazzi \& Ruzmaikin (2004) discussed a linear solar dynamo model with an ad hoc nonaxisymmetric $m=1$ contribution to the alpha-effect, but without the latitudinal oscillations that are included in our experiment. Unsurprisingly, they also found a nonaxisymmetric mode to be excited (see also Moss et al. 1991b).

Again, explanations such as these exclude true differential rotation of active longitudes, but are compatible with a stroboscopic effect.

\subsection{Polar migration}

The Earth's poloidal magnetic field appears to reverse in a way that is rather different to that usually resulting from solar dynamo models, in that the Northen magnetic pole migrates from somewhere near the Northern geographic pole to the vicinity of the Southern. It has been claimed (see e.g. Ermakov et al. 1995) that the Sun's poloidal field reverses in a similar fashion, and that the path of the magnetic poles traces the active longitudes. This appears an attractive idea, but does not directly address any alternation of active longitudes, nor their persistence outside the episodes of migration. Further, butterfly diagrams for the poloidal field suggest that the component of largest scale exhibits standing wave behaviour, with a node near the equator (Obridko et al. 2005) - this appears inconsistent with the above hypothesis.

\section{Active longitudes in stellar data}

As it is natural to expect, the phenomenon of active longitudes is not specific to the Sun. Here we briefly summarize the available knowledge concerning stellar active longitudes.

Binary systems intrinsically violate axisymmetry and obviously provide additional mechanisms to excite nonaxisymmetric magnetic field structures which are not available to single stars (Moss \& Tuominen 1997; Moss et al. 2002; Holzwarth $\&$ Schüssler 2003). Indeed, decades of continuous photometric monitoring of RS CVn-type binaries revealed that large active regions maintain their identity for years and indicate the presence of one or two active longitudes (Berdyugina \& Tuominen 1998; Lanza et al. 1998; Rodonò et al. 2000). However, some single rapidly rotating stars, such as FK Comtype stars and young solar analogues, also demonstrate active longitudes (Jetsu et al. 1993, 1999; Berdyugina et al. 2002; Korhonen et al. 2002; Berdyugina \& Järvinen 2005).

A common feature of stellar active longitudes is their migration with respect to the orbital reference frame in binaries or relative to a long-term average rotation rate in single stars. Moreover, variations of the migration rate over a stellar cycle are more prominent in single stars than in binaries, suggesting a stronger differential rotation in single stars. Also, the migration pattern of the active longitudes in young solar analogues is very similar to that observed on the Sun and indicate solar-type cycles on these stars (Berdyugina 2004). Although active longitudes endure for a long time, the active regions they consist of evolve in size. For instance, while one active longitude reduces its activity level, the other increases, which suggests a redistribution of the spotted area between the opposite hemispheres. When the active longitudes have about the same activity level a switch of the dominant activity from one longitude to the opposite one occurs. Such a phenomenon was first observed on FK Com (Jetsu et al. 1993) and was tentatively called a "flip-flop". Later it was discovered that these flip-flops recur regularly and thus indicate a new type of stellar cycle which is related to active longitudes, i.e. a flip-flop cycle (Berdyugina \& Tuominen 1998; Korhonen et al. 2002; Berdyugina \& Järvinen 2005). Of particular interest is that a flip-flop cycle of about 3.7 years was also revealed in the evolution of the spot area on the Sun (Berdyugina \& Usoskin 2003). The frequency ratio of the sunspot-like cycle and the flip-flop cycle appears to be different for binary components and single stars. In RS CVn-type stars exhibiting both types of 
cycles, flip-flops appear to occur at the frequency of the spottedness cycle, while in young dwarfs and the Sun flipflop cycles are 3-4 times shorter than the sunspot-like cycle (Berdyugina 2004). Note, however, that the sample of stars being known to exhibit persistent nonaxisymmetric spot distributions and flip-flop cycles is limited to about a dozen and includes only rapidly rotating stars at different stages of evolution. Thus, although the phenomenon appears similar in different stars, the underlying excitation mechanisms may still differ between for instance, single and binary stars. Rapid rotation resulting in enhanced magnetic activity can also influence the internal rotation law and the excitation of nonaxisymmetric dynamo modes.

The switching of active longitudes and flip-flop cycles observed on the Sun and other active stars, contrary perhaps to first impressions, does not necessarily imply the existence of oscillating nonaxisymmetric fields. Coexistence of oscillating axisymmetric and steady nonaxisymmetric modes can result in the appearance of flip-flop cycles. The possibility of such a mechanism was first demonstrated by the mean-field dynamo calculation of Moss (2004) who obtained a stable solution with an oscillating axisymmetric mode that varies between almost dipole-like and almost quadrupole-like parities and a steady, mixed-polarity nonaxisymmetric mode. In this case flip-flops are quasi-periodic and as frequent as sign changes of the axisymmetric mode, which is reminiscent of the behaviour observed in some RS CVn stars. More frequent flip-flops, compared to the sunspot-like cycle in single stars and the Sun, suggest a more complex field configuration. Fluri \& Berdyugina (2004) showed that flip-flops could also occur due to alternation of relative strengths of two nonaxisymmetric modes without sign changes of any of the modes involved, but dynamo theorists have yet to produce such configurations. If in addition a co-existing axisymmetric mode were changing its sign with a different frequency, it would result in the behaviour observed in solar-type stars.

Note that in principle a regular flip-flop cycle could be associated with the combination of a steady relic field and an oscillating axisymmetric field generated by a dynamo - for example, a combination of steady S1 and oscillating A0 structures (e.g. Fluri \& Berdyugina 2004; Moss 2004). However in the solar context, this explanation appears implausible inter alia because of the observed rotation rate of the nonaxisymmetric structures - see the discussion in Sect. 4.3.

\section{Conclusion}

We conclude that the mechanisms discussed above for generating active longitudes can be viable if the stroboscopic interpretation is accepted. A quantitative description of the phenomenon however needs specific numerical modelling this will necessitate a detailed and quite sophisticated effort. It is difficult to exclude a priori an MHD-based explanation for the active longitude that assumes the presence of true differential rotation. Such an explanation should include a nonaxisymmetric magnetic structure with solid body rotation somehow produced deep below the solar surface. The surface nonaxisymmetry have to be associated with a magnetic field that separates from this magnetic structure and is strongly affected by differential rotation in the solar convection zone. Our attempts to find such a solution in the framework of the ideas discussed above were unsuccessful. Accordingly, we consider this possibility as much less probable than a stroboscopic interpretation of the kind discussed in Sect. 3.

Acknowledgements. We thank Axel Brandenburg for his comments. The Academy of Finland is acknowledged for financial support through grants 43039, 107796. D.S. and D.M. are grateful to the University of Oulu for hospitality, and D.S. also acknowledges financial support from RFBR (grant 04-02-16068). D.S. and D.M. were visitors at the Isaac Newton Institute, University of Cambridge, where they first discussed some of the ideas presented in this paper.

\section{References}

Bai, T. 2003, ApJ, 585, 1114

Balthasar, H., \& Schüssler, M. 1983, Sol. Phys., 87, 23

Balthasar, H., Vazquez, M., \& Woehl, H. 1986, A\&A, 155, 87

Barker, D. M., \& Moss, D. 1994, A\&A, 283, 1009

Bassom, A., Kuzanyan, K., Soward, A. M., \& Sokoloff, D. 2005, Geophys. Astrophys. Fluid Dyn., 99, 309

Benevolenskaya, E. E., Kosovichev, A. G., \& Scherrer, P. H. 1999a, Sol. Phys., 190, 145

Benevolenskaya, E. E., Hoeksema, J. T., Kosovichev, A. G., \& Scherrer, P. H. 1999b, ApJ, 517, L163

Berdyugina, S. V. 2004, Sol. Phys., 224, 123

Berdyugina, S. V., \& Tuominen, I. 1998, A\&A, 336, L25

Berdyugina, S. V., Pelt, J., \& Tuominen, I. 2002, A\&A, 394, 505

Berdyugina, S. V., \& Usoskin, I. G. 2003, A\&A, 405, 1121

Berdyugina, S. V., \& Järvinen, S. P. 2005, AN, 326, 283

Bigazzi, A., \& Ruzmaikin, A. 2004, ApJ, 604, 944

Boyer, D. W., \& Levy, E. H. 1984, ApJ, 277, 845

Bobova, V. P., \& Stepanian, N. N. 1994, Sol. Phys., 152, 291

Brandenburg, A. 2005, ApJ, 625, 539

Bravo, S., \& Stewart, G. A. 1996, Adv. Sp. Res., 17(4-5), 221

Bumba, V. 1991, Bull. Astron. Inst. Czechosl., 42, 381

Bumba, V., \& Howard, R. 1969, Sol. Phys., 7, 28

Bumba, V., \& Obridko, V. N. 1969, Sol. Phys., 6, 104

Carrington, R. C. 1863, Observations of the Spots on the Sun

Chidambara, A. 1932, MNRAS, 93, 150

Dicke, R. H. 1979, ApJ, 228, 898

Dikpati, M., Cally, P. S., \& Gilman, P. 2004, ApJ, 610, 597

Ermakov, F. A., Obridko, V. N., \& Shelting, B. D. 1995, Astron. Rep., 39,672

Esltner, D., \& Korhonen, H. 2005, Astron. Nachr., 326, 278

Fluri, D. M., \& Berdyugina, S. V. 2004, Sol. Phys., 224, 153

Fluri, D. M., \& Berdyugina, S. V. 2005, in 22nd NSO Workshop Large Scale Structures and their Role in Solar Activity, ed. K. Sankarasubramanian, M. Penn, \& A. Pevtsov, ASP Conf. Ser., 346, 167

Goode, P. R., \& Thompson, M. J. 1992, ApJ, 395, 307

Holzwarth, V., \& Schüssler, M. 2003, 405, 303

Jetsu, L., Pelt, J., \& Tuominen, I. 1993, A\&A, 278, 449

Jetsu, L., Pohjolainen, S., Pelt., J., \& Tuominen, I. 1997, A\&A, 318, 293

Jetsu, L., Pelt, J., \& Tuominen, I. 1999, A\&A, 351, 212

Kitchatinov, L. L., Jardine, M., \& Collier Cameron, A. 2001, A\&A, 374,250

Knaack, R., Stenflo, J. O., \& Berdyugina, S. 2004, A\&A, 418, L17 
Korhonen, H., Berdyugina, S. V., \& Tuominen, I. 2002, A\&A, 390, 179

Korhonen, H., \& Elstner, D. 2005, A\&A, in press

Lanza, A. F., Catalano, S., Cutispoto, G., Pagano, I., \& Rodonò, M. 1998, A\&A, 332, 541

Lee, J. S. 1986, Opt. Engineering 25(5), 636

Lopez Arroyo, M. 1961, The Observatory, 81, 205

Mason, J., Hughes, D. W., \& Tobias, S. M. 2002, ApJ, 580, L89

Mordvinov, A. V., \& Kitchatinov, L. L. 2004, Astron. Rep., 48, 254

Mestel, L. 1961, MNRAS, 122, 473

Mestel, L., \& Weiss, N. O. 1987, MNRAS, 226, 123

Moss, D. 1999, MNRAS, 306, 300

Moss, D. 2004, MNRAS, 352, L17

Moss, D. 2005, A\&A, 432, 249

Moss, D., \& Brooke, J. 2000, MNRAS, 315, 521

Moss, D., \& Tuominen, I. 1997, A\&A, 321, 151

Moss, D., Tuominen, I., \& Brandenburg, A. 1991a, A\&A, 245, 129

Moss, D., Brandenburg, A., \& Tuominen, I. 1991b, A\&A, 247, 576

Moss, D., Barker, D. M., Brandenburg, A., \& Tuominen, I. 1995, A\&A, 284, 155

Moss, D., Piskunov, N., \& Sokoloff, D. 2002, A\&A, 396, 885

Neugebauer, M., Smith, E. J., Ruzmaikin, A., Feynman, J., \& Vaughan, A. H. 2000, J. Geophys. Res., 105, 2315
Obridko, V. N., Kuzanyan, K. M., Shelting, B. D., Sokoloff, D. D., \& Zakharov, V. G. 2005, Proc. JENAM Meeting The Many Scales in the Universe, in press

Parker, E. 1955, ApJ, 122, 293

Pelt, J., Tuominen, I., \& Brooke, J. 2005, A\&A, 429, 1093

Pulkkinen, P. J., Brooke, J., Pelt, J., \& Tuominen, I. 1999, A\&A, 341, 43

Pudovkin, M. I., \& Benevolenskaya, E. E. 1982, Sov. Astron. Lett., 8, 273

Rädler, K.-H. 1986, in Plasma Physics, ESA SP-251, 569

Rädler, K.-H., Weidemann, E., Meinel, R., Brandenburg, A., \& Tuominen, I. 1990, A\&A, 239, 413

Roberts, P. H., \& Stix, M. 1972, A\&A, 18, 453

Rodonò, M., Messina, S., Lanza, A. F., Cutispoto, G., \& Teriaca, L. 2000, A\&A, 358, 624

Ruzmaikin, A. A. 1998, Sol. Phys., 181, 1

Schou, J., Antia, H. M., Basu, S., et al. 1998, ApJ, 505, 390

Stewart, G. A., \& Bravo, S. 1996, Adv. Sp. Res., 17(4-5), 217

Usoskin, I. G., Berdyugina, S. V., \& Poutanen, J. 2005, A\&A, 441, 347

Vitinskij, Yu. I. 1969, Sol. Phys., 7, 210

Warwick, C. S. 1965, ApJ, 141, 500 